\title{
ON SOME DIOPHANTINE EQUATIONS
}

\author{
S. BUJAČIĆ BABIĆ AND K. NABARDI
}

Received 29 May, 2018

\begin{abstract}
In this paper we deal with some Diophantine equations and present infinitely many positive integer solutions for each one of them.
\end{abstract}

2010 Mathematics Subject Classification: 11D45; 11D25

Keywords: Diophantine equation

\section{INTRODUCTION}

The Diophantine equations

$$
x^{n}+y^{n}=u^{n}+v^{n}, \quad n=2,3,4
$$

have been considered by many mathematicians. The case $n=2$ was presented in $[13,14]$ while Euler [8] and Binet [3] considered the case $n=3$. Parametric solutions of the above equation for $n=4$ can be found in $[4,10]$. Some researches considered more general Diophantine equations with more variables or with integer coefficients that are not all equal to $1[5-7,11,12]$.

In this paper, we deal with the equations

$$
\begin{aligned}
& X^{3}-Y^{2}=X^{2}-Z^{3}, \\
& X^{3} \pm Y^{3}=U^{4}-V^{4}
\end{aligned}
$$

and obtain infinitely many positive integer solutions for each one of them. We consider the equation of the form

$$
A m^{4}+B n^{4}+C p^{4}+D q^{4}=A r^{4}+B s^{4}+C t^{4}+D u^{4}, \quad A, B, C, D \in \mathbb{Z},
$$

introduce some linear transformations and set some special conditions on its coefficients. Some recent papers deal with the similar problems. In [9] the authors investigate Diophantine equations of the form

$$
T^{2}=G(\bar{X}), \quad \bar{X}=\left(X_{1}, X_{2}, \cdots, X_{m}\right),
$$

The first author is supported by the Croatian Science Foundation under the project no. IP-2018-011313.

The second author is supported by Iran National Science Foundation grant number 95005149 . 
where $m=3$ or $m=4$ and $G$ is a specific homogenous quintic form. The equations

$$
a\left(X_{1}^{\prime 5}+X_{2}^{\prime 5}\right)+\sum_{i=0}^{n} a_{1} X_{i}^{5}=b\left(Y_{1}^{\prime 3}+Y_{2}^{\prime 3}\right)+\sum_{i=0}^{n} b_{i} Y_{i}^{3}
$$

where $m, n \in \mathbb{N} \cup\{0\}$ and $a, b \neq 0, a_{i}, b_{i}$ are fixed arbitrary rational numbers are studied in [2]. The theory of elliptic curves is used in order to solve (1.2) which is transformed to a cubic or a quartic elliptic curve with a positive rank. In [1, Main Theorem 2] authors prove that

$$
\sum_{i=1}^{n} p_{i} \cdot x_{i}^{a_{i}}=\sum_{j=1}^{m} q_{j} \cdot y_{j}^{b_{j}}
$$

$m, n, a_{i}, b_{j} \in \mathbb{N}, p_{i}, q_{j} \in \mathbb{Z}, i=1, \ldots, n, j=1, \ldots, m$ has a parametric solution and infinitely many solutions in nonzero integers if there exists an $i$ such that $p_{i}=1$ and $\left(a_{i}, a_{1} a_{2} \cdots a_{i-1} a_{i+1} \cdots a_{n} b_{1} b_{2} \cdots b_{m}\right)=1$ or there exists a $j$ such that $q_{j}=1$ and $\left(b_{j}, a_{1} \cdots a_{n} b_{1} \cdots b_{j-1} b_{j+1} \cdots b_{m}\right)=1$. In this article, even though linear transformations are also used, we introduce a different approach and some different conditions on the integer coefficients in order to solve (1.1).

$$
\text { 2. EQuATION } X^{3}-Y^{2}=X^{2}-Z^{3}
$$

For start, we deal with the Diophantine equation

$$
X^{3}-Y^{2}=X^{2}-Z^{3}
$$

It is easily shown that equation (2.1) has infinitely many solutions $(X, Y, Z)=\left(1, n^{3}, n^{2}\right), n \in \mathbb{N}$. The main task of our work in this section is to discover whether there are more positive integer solutions of (2.1). We set

$$
c=x^{2}, \quad d=y^{2}
$$

and obtain

$$
\begin{aligned}
c^{3}+d^{3}=x^{6}+y^{6}=\left(x^{3}-(y i)^{3}\right) & \left(x^{3}+(y i)^{3}\right) \\
& =(x-y i)\left(x^{2}+x y i-y^{2}\right)(x+y i)\left(x^{2}-x y i-y^{2}\right) .
\end{aligned}
$$

If we define

$$
a-b i=(x+y i)\left(x^{2}+x y i-y^{2}\right) \quad \text { and } \quad a+b i=(x-y i)\left(x^{2}-x y i-y^{2}\right),
$$

we get

$$
c^{3}+d^{3}=a^{2}+b^{2}
$$

for $a=x^{3}-2 y^{2} x$ and $b=y^{3}-2 x^{2} y$. From (2.2) we obtain the equation

$$
\left(x^{2}\right)^{3}+\left(y^{2}\right)^{3}=\left(x^{3}-2 y^{2} x\right)^{2}+\left(y^{3}-2 x^{2} y\right)^{2} .
$$

First we deal with the case

$$
y^{2}=y^{3}-2 x^{2} y, \quad y \neq 0
$$


and get

$$
(2 y-1)^{2}-8 x^{2}=1
$$

After introducing $y^{\prime}=2 y-1$ in (2.3) a Pell equation

$$
y^{\prime 2}-8 x^{2}=1
$$

is obtained. Some of its solutions are $\left(y^{\prime}, x\right)=\{(3,1),(17,6),(99,35), \ldots\}$. Finally, solutions of equation (2.3) are

$$
(x, y)=\{(1,2),(6,9),(35,50), \ldots\} .
$$

The inequality $x<y$ implies $x^{3}-2 y^{2} x<0$, so $Y<0$. Because we deal with equation of the form (2.1), we take $|Y|$ and get infinitely many positive integer solutions

$$
(X, Y, Z)=\left(y^{2}, x^{3}-2 y^{2} x, x^{2}\right)=\left\{(4,7,1),(81,756,36),\left(50^{2}, 132125,35^{2}\right), \ldots\right\}
$$

of (2.1).

Alternatively, we get

$$
8 y^{2}=\frac{8 x^{3}}{2 x+1}
$$

for $y^{2}=x^{3}-2 y^{2} x$. Obviously, $\frac{8 x^{3}}{2 x+1} \in \mathbb{N}$ if and only if $(2 x+1) \mid 1$ which happens for only $2 x+1=1$ which implies $x=0, y=0$. This case is not considered. Cases $x^{2}=x^{3}-2 y^{2} x$ and $x^{2}=y^{3}-2 x^{2} y$ do not provide us with new solutions. Consequently, (2.1) has infinitely many positive integer solutions of the form

$$
(X, Y, Z)=\left(y^{2}, x^{3}-2 y^{2} x, x^{2}\right),
$$

where $(x, y)$ are solutions of equation (2.3).

Remark 1. It can be noticed that for $X=Z$ equation (2.1) becomes

$$
X^{2}(2 X-1)=Y^{2},
$$

so $X=Z=2 k^{2}+2 k+1, k \in \mathbb{N}$ will provide a solution. This approach can be generalized by taking $X=m Z, Y=n Z$ for $m, n \in \mathbb{N}$. We get

$$
Z=\frac{m^{2}+n^{2}}{m^{3}+1}
$$

and by fixing $m$ we may yield some solutions. For example, if $m=4$, then $n \equiv 7,32,33,58(\bmod 65)$ will provide solutions. Similarily, for $m=9$, we obtain that $n \equiv 97,243,487,630(\bmod 730)$ will provide solutions. This approach works if $m$ is a square, however we also have solutions for $m=28$. Therefore, it may be difficult to completely classify all the solutions here. 


\section{EQUATION $X^{3}+Y^{3}=U^{4}-V^{4}$}

In this section we deal with the equation

$$
X^{3}+Y^{3}=U^{4}-V^{4}
$$

It is easy to notice that $(X, Y, U, V)=(m,-m, n, n)$ is a trivial solution of (3.1) for $m, n \in \mathbb{N}$, while the smallest nontrivial solution of (3.1) is $(X, Y, U, V)=(4,1,3,2)$.

Two different linear transformations are considered and for each one of them we give a different class of infinitely many positive integer solutions of equation (3.1).

\subsection{The First Method}

Let

$$
X=p x+u, \quad Y=q x-u, \quad U=x+v, \quad V=p x-v,
$$

$p, q, u, v \in \mathbb{N}$. Introducing (3.2) into the initial equation (3.1), we get

$$
\alpha x^{4}+\beta x^{3}+\gamma x^{2}+\delta x=0,
$$

where

$$
\begin{array}{ll}
\alpha=p^{4}-1, & \beta=p^{3}+q^{3}-4 v-4 p^{3} v, \\
\gamma=3 p^{2} u-3 q^{2} u-6 v^{2}+6 p^{2} v^{2}, & \delta=3 p u^{2}+3 q u^{2}-4 v^{3}-4 p v^{3} .
\end{array}
$$

For $\delta=0$ in (3.4), we obtain

$$
3(p+q) u^{2}=4(p+1) v^{3} .
$$

Additionally, we set $u=t^{3}, v=t^{2}$ and get $p=3 q-4$. Finally, the following is obtained

$$
\begin{aligned}
& \alpha=81 q^{4}-432 q^{3}+864 q^{2}-768 q+255 \\
& \beta=432 q^{2} t^{2}-576 t^{2} q-108 q^{2}+252 t^{2}-108 q^{3} t^{2}+28 q^{3}+144 q-64, \\
& \gamma=24 q^{2} t^{3}+54 q^{2} t^{4}-72 q t^{3}-144 q t^{4}+90 t^{4}+48 t^{3} .
\end{aligned}
$$

Let

$$
\gamma=6 t^{3}(q-1)(4 q+9 q t-15 t-8)=0 .
$$

In that case, we have $q=\frac{15 t+8}{9 t+4}$ and therefore (3.3) becomes

$$
\begin{aligned}
& \frac{11664 t^{3}+23328 t^{2}+16128 t+3840}{(9 t+4)^{4}} x^{4}+ \\
& +\frac{-52488 t^{6}-128304 t^{5}-87480 t^{4}+27216 t^{3}+61632 t^{2}+27648 t+4096}{(9 t+4)^{4}} x^{3}=0 .
\end{aligned}
$$

We get

$$
x=\frac{(9 t+4)\left(243 t^{4}+324 t^{3}-27 t^{2}-192 t-64\right)}{6\left(81 t^{2}+108 t+40\right)}
$$


and

$$
\begin{aligned}
& X=\frac{2673 t^{5}+5508 t^{4}+2589 t^{3}-1944 t^{2}-2112 t-512}{6\left(81 t^{2}+108 t+40\right)}, \\
& Y=\frac{3159 t^{5}+6156 t^{4}+1947 t^{3}-3096 t^{2}-2496 t-512}{6\left(81 t^{2}+108 t+40\right)}, \\
& U=\frac{2187 t^{5}+4374 t^{4}+1701 t^{3}-1596 t^{2}-1344 t-256}{6\left(81 t^{2}+108 t+40\right)}, \\
& V=\frac{2187 t^{5}+4374 t^{4}+1701 t^{3}-2184 t^{2}-2112 t-512}{6\left(81 t^{2}+108 t+40\right)} .
\end{aligned}
$$

After eliminating denominators in (3.5), we have

$$
\begin{aligned}
& X=216\left(81 t^{2}+108 t+40\right)^{3}\left(2673 t^{5}+5508 t^{4}+2589 t^{3}-1944 t^{2}-2112 t-512\right), \\
& Y=216\left(81 t^{2}+108 t+40\right)^{3}\left(3159 t^{5}+6156 t^{4}+1947 t^{3}-3096 t^{2}-2496 t-512\right), \\
& U=36\left(81 t^{2}+108 t+40\right)^{2}(9 t+8)\left(243 t^{4}+270 t^{3}-51 t^{2}-132 t-32\right), \\
& V=36\left(81 t^{2}+108 t+40\right)^{2}\left(2187 t^{5}+4374 t^{4}+1701 t^{3}-2184 t^{2}-2112 t-512\right) .
\end{aligned}
$$

For $t=1$, the solutions of (3.1) are

$$
(X, Y, U, V)=(16087625952048,13379550896592,9563979816,65207230704) \text {, }
$$

while $t=2$ leads us to

$$
(X, Y, U, V)=(7664511333888000,8313869044224000,
$$

$1746900979200,1696715481600)$.

We get a positive integer solution $(X, Y, U, V)$ of equation (3.1) for every $t \in \mathbb{N}$. So, the presented method generates infinitely many positive integer solutions of the initial equation (3.1).

\subsection{The Second Method}

Again, we deal with (3.1) and start this new method by introducing a different linear transformation in order to reach more (different) positive integer solutions. Let

$$
X=u, \quad Y=q x-u, \quad U=x+u, \quad V=p x+u,
$$

$p, q, u \in \mathbb{N}$. Like in the previous subsection, introducing these linear transformations into (3.3), leads us to the expression of the form

$$
A x^{4}+B x^{3}+C x^{2}+D x=0,
$$


where

$$
\begin{array}{llrl}
A & =p^{4}-1, & B & =q^{3}+4 p^{3} u-4 u, \\
C & =6 p^{2} u^{2}-6 u^{2}-3 q^{2} u, & D & =4 u^{3} p-4 u^{3}+3 u^{2} q .
\end{array}
$$

We obtain $u=\frac{3 q}{4(1-p)}, p \neq 1$ for $D=0$. After introducing the latter expression into (3.8) and canceling the denominators we get

$A=8 p^{5}-8 p^{4}-8 p+8, \quad B=8 q^{3} p-24 p^{3} q-8 q^{3}+24 q, \quad C=27 q^{2} p+18 q^{3}+27 q^{2}$.

We obtain $q=-\frac{3}{2}(p+1)$ for $C=0$. Under those conditions we get

$$
A=p^{4}-1, \quad B=\frac{9}{8}(p+1)(p-1)^{2}
$$

and (3.7) becomes $\left(p^{4}-1\right) x^{4}+\frac{9}{8}(p+1)(p-1)^{2} x^{3}=0$ where

$$
x=\frac{9(1-p)}{8\left(p^{2}+1\right)} .
$$

After plugging all of these results into (3.6) and canceling the denominators, we finally get

$$
\begin{aligned}
& X=18(p-1)^{3}(p+1)\left(p^{2}+1\right)^{4}, \\
& Y=9(p+1)\left(p^{2}-6 p+1\right)(p-1)^{3}\left(p^{2}+1\right)^{3}, \\
& U=9 p\left(p^{2}+3\right)(p-1)^{2}\left(p^{2}+1\right)^{2}, \\
& V=9\left(3 p^{2}+1\right)\left(p^{2}+1\right)^{2}(p-1)^{2} .
\end{aligned}
$$

Remark 2. According to (3.9), $X>0$ is satisfied for $p \in \mathbb{Z} \backslash\{0,1\}$. Also, $Y>0$ is satisfied for $p \in \mathbb{Z} \backslash\{-1,0,1,2,3,4,5\}$. Therefore, by the introduced method we are again able to generate infinitely many positive integer solutions of (3.1).

$$
\text { 4. The EQuATion } X^{3}-Y^{3}=U^{4}-V^{4}
$$

In this section we deal with the equation

$$
X^{3}-Y^{3}=U^{4}-V^{4} .
$$

If we introduce $Y \rightarrow-Y$ or $X \rightarrow-X$ in (3.1), it is clear that one gets solutions of (4.1), but, as we mentioned earlier, we are interested only in positive integer solutions. Therefore, we consider (3.1) and (4.1) as two different equations.

Clearly, $(X, Y, U, V)=\left(1, n^{4}, 1, n^{3}\right), n \in \mathbb{N}$ are trivial solutions of (4.1).

After introducing linear transformations

$$
X=p x+u, \quad Y=q x+u, \quad U=r x+u, \quad V=u,
$$

$p, q, r, u \in \mathbb{N}$ into (4.1), we get the equation

$$
M x^{4}+N x^{3}+P x^{2}+Q x=0,
$$


where

$$
\begin{aligned}
M & =-r^{4}, & N & =p^{3}-q^{3}-4 r^{3} u, \\
P & =3 p^{2} u-3 q^{2} u-6 r^{2} u^{2}, & Q & =3 u^{2} p-3 u^{2} q-4 u^{3} r .
\end{aligned}
$$

If we set $Q=0$, it is easily obtained $u=\frac{3(p-q)}{4 r}$. Plugging this new form of $u$ into (4.2), we get

$$
M=-r^{4}, \quad N=(p-q)\left(p^{2}+p q-3 r^{2}+q^{2}\right), \quad P=\frac{9(p-q)^{2}(2 p+2 q-3 r)}{8 r} .
$$

We obtain $r=\frac{2}{3}(p+q)$ for $2 p+2 q-3 r=0$. So, introducing the above expressions into (4.3), we get

$$
\begin{gathered}
-\frac{16}{81}(p+q)^{4} x^{4}-\frac{1}{3}(p-q)\left(p^{2}+5 p q+q^{2}\right) x^{3}=0, \\
x=-\frac{27}{16} \frac{(p-q)\left(p^{2}+5 p q+q^{2}\right)}{(p+q)^{4}} .
\end{gathered}
$$

Finally, we define $p^{2}+5 p q+q^{2}-16=0$ which is a quadratic equation in $p$. So,

$$
p=\frac{-5 q \pm \sqrt{21 q^{2}+64}}{2} \text {. }
$$

Let $21 q^{2}+64=r^{2}, r \in \mathbb{Z}$. Solution $(q, r)=(0,8)$ is a trivial solution for this equation. Therefore, considering $r=m q+8$ and $21 q^{2}+64=r^{2}$ leads us to $q=\frac{16 m}{21-m^{2}}$ and $p=\frac{4\left(m^{2}+10 m+21\right)}{m^{2}-21}$ or $p=\frac{4\left(m^{2}-10 m+21\right)}{21-m^{2}}$. We get

$$
x=\frac{-27\left(m^{2}-21\right)^{3}\left(m^{2}+14 m+21\right)}{64\left(m^{2}+6 m+21\right)^{4}}
$$

for $p=\frac{4\left(m^{2}+10 m+21\right)}{m^{2}-21}$. After canceling the denominators, we obtain

$$
\begin{aligned}
& X=-9\left(m^{2}+14 m+21\right)\left(m^{6}-6 m^{5}-405 m^{4}-3204 m^{3}-8505 m^{2}-2646 m+9261\right), \\
& Y=18\left(m^{2}+14 m+21\right)\left(m^{6}+24 m^{5}+171 m^{4}+720 m^{3}+3591 m^{2}+10584 m+9261\right), \\
& U=27 \cdot 2^{2} \cdot m\left(m^{2}+14 m+21\right)\left(m^{2}+10 m+21\right) \\
& V=9\left(m^{2}+6 m+21\right)^{2}\left(m^{2}+14 m+21\right)
\end{aligned}
$$

Some elementary analysis leads us to conclusion that $X, Y>0$ for every $m \in \mathbb{N}$. The described method generates infinitely many positive integer solutions $(X, Y, U, V)$ for the initial equation (4.1). Some of them are introduced in the following table.

\begin{tabular}{c||c||c||c||c}
$m$ & $X$ & $Y$ & $U$ & $V$ \\
\hline 1 & 1783296 & 15780096 & 124416 & 254016 \\
\hline 2 & 29712807 & 51631434 & 515160 & 653013 \\
\hline 3 & 126531072 & 146686464 & 1399680 & 1492992 \\
\hline 4 & 375132519 & 380970594 & 3093552 & 3114477 \\
\hline 5 & 911771136 & 917584128 & 6013440 & 6030144
\end{tabular}


Remark 3. If we apply the previous process by taking $p=\frac{4\left(m^{2}-10 m+21\right)}{21-m^{2}}$, no new solutions are obtained.

Some basic calculations give us $X, Y>0$ for $m \leq-1$ and these solutions are already obtained for $m>0$ in (4.4). This is shown in the following table. It is useful to notice that, even though we get integer solutions in this case, we do not consider negative ones because $U, V$ are introduced with even powers in (4.1).

\begin{tabular}{c||c||c||c||c}
$m$ & $X$ & $Y$ & $U$ & $V$ \\
\hline-1 & 1783296 & 15780096 & -124416 & 254016 \\
\hline-2 & 29712807 & 51631434 & -515160 & 653013 \\
\hline-3 & 126531072 & 146686464 & -1399680 & 1492992 \\
\hline-4 & 375132519 & 380970594 & -3093552 & 3114477 \\
\hline-5 & 911771136 & 917584128 & -6013440 & 6030144
\end{tabular}

5. The EQUATION $A m^{4}+B n^{4}+C p^{4}+D q^{4}=A r^{4}+B s^{4}+C t^{4}+D u^{4}$

We deal with the equation of the form

$$
A m^{4}+B n^{4}+C p^{4}+D q^{4}=A r^{4}+B s^{4}+C t^{4}+D u^{4}, \quad A, B, C, D \in \mathbb{Z} .
$$

Let

$$
\begin{aligned}
& m=l x+1, \\
& n=5 x+k, \\
& p=4 x+1, \\
& q=2 x, \\
& r=l x-1, \quad s=4 x-k, \\
& t=5 x-1, \\
& u=x \text {, }
\end{aligned}
$$

for $k, l \in \mathbb{N}$. Introducing these linear transformations into (5.1), one gets the equation of the form

$$
a x^{4}+b x^{3}+c x^{2}+d x=0
$$

where

$$
\begin{array}{ll}
a=-396 C+15 E+369 B, & b=756 B k+8 A l^{3}+756 C, \\
c=-54 C+54 B k^{2}, & d=8 A l+36 B k^{3}+36 C .
\end{array}
$$

We set the conditions $c=d=0$ and get

$$
C=B k^{2} \quad \text { and } \quad l=-\frac{9}{2} \cdot \frac{B k^{3}+C}{A} .
$$

Introducing (5.3) into (5.2) leads us to

$$
\begin{aligned}
& -\frac{3\left(-123 B A^{2}+123 B k^{2} A^{2}-5 E A^{2}\right) x^{4}}{A^{2}} \\
& -\frac{3\left(729 B^{3} k^{7}+243 B^{3} k^{6}+243 B^{3} k^{9}+729 B^{3} k^{8}-252 B k^{2} A^{2}-252 B A^{2} k\right) x^{3}}{A^{2}}=0
\end{aligned}
$$


and

$$
x=\frac{9 B k\left(-27 B^{2} k^{8}-81 B^{2} k^{7}-81 B^{2} k^{6}-27 B^{2} k^{5}+28 A^{2}+28 k A^{2}\right)}{A^{2}\left(123 B k^{2}-5 E-123 B\right)} .
$$

After plugging all results into (5.3) and cancelling the denominators, we get

$$
\begin{aligned}
m= & 2187 B^{4} k^{12}+8748 B^{4} k^{11}+13122 B^{4} k^{10}+8748 B^{4} k^{9} \\
& -4536 B^{2} k^{4} A^{2}-2268 B^{2} k^{5} A^{2}+2187 B^{4} k^{8}-2268 B^{2} k^{3} A^{2} \\
& +246 A^{3} B k^{2}-10 A^{3} E-246 A^{3} B \\
n= & 2 A k\left(-1215 B^{3} k^{8}-3645 B^{3} k^{7}-3645 B^{3} k^{6}-1215 B^{3} k^{5}\right. \\
& \left.+1137 B A^{2}+1260 B k A^{2}+123 A^{2} B k^{2}-5 A^{2} E\right), \\
p=2 A\left(-972 B^{3} k^{9}-2916 B^{3} k^{8}-2916 B^{3} k^{7}-972 B^{3} k^{6}\right. & \\
& \left.+1008 B k A^{2}+1131 A^{2} B k^{2}-5 A^{2} E-123 B A^{2}\right), \\
q= & 36 A B k\left(-27 B^{2} k^{8}-81 B^{2} k^{7}-81 B^{2} k^{6}-27 B^{2} k^{5}+28 A^{2}+28 k A^{2}\right), \\
r= & -2187 B^{4} k^{12}-8748 B^{4} k^{11}-13122 B^{4} k^{10}-8748 B^{4} k^{9} \\
& +4536 B^{2} k^{4} A^{2}+2268 B^{2} k^{5} A^{2}-2187 B^{4} k^{8}+2268 B^{2} k^{3} A^{2} \\
& +246 A^{3} B k^{2}-10 A^{3} E-246 A^{3} B, \\
s= & A k\left(972 B^{3} k^{8}+2916 B^{3} k^{7}+2916 B^{3} k^{6}+972 B^{3} k^{5}-1131 B A^{2}\right. \\
& \left.-1008 B k A^{2}+123 A^{2} B k^{2}-5 A^{2} E\right), \\
t= & 2 A\left(-1215 B^{3} k^{9}-1645 B^{2} k^{8}-1645 B^{3} k^{7}-1215 B^{3} k^{6}+1260 B k A^{2}\right. \\
& \left.+1137 A^{2} B k^{2}+5 A^{2} E+123 B A^{2}\right), \\
u=18 A B k\left(-27 B^{2} k^{8}-81 B^{2} k^{7}-81 B^{2} k^{6}-27 B^{2} k^{5}+28 A^{2}+28 k A^{2}\right) . & \\
&
\end{aligned}
$$

\subsection{Numerical examples}

We solve equation (5.1) of the form

$$
m^{4}+2 n^{4}+2 p^{4}+5 q^{4}=r^{4}+2 s^{4}+2 t^{4}+5 u^{4}
$$

where $A=1, B=2, C=2, D=5$, so $k=1$. According to (5.4), we obtain

$$
\begin{aligned}
& m=523534, \quad n=145490, \quad \quad p=116402, \quad q=58176, \\
& r=523634, \quad s=116302, \quad t=145390, \quad u=39088
\end{aligned}
$$

or

$$
\begin{aligned}
& m=261767, \quad n=72745, \quad \quad p=58201, \quad q=29088 \text {, } \\
& r=261817, \quad s=58151, \quad t=72695, \quad u=14544 \text {. }
\end{aligned}
$$

Let

$$
3 m^{4}+2 n^{4}+8 p^{4}+5 q^{4}=3 r^{4}+2 s^{4}+8 t^{4}+5 u^{4} .
$$


We have

$$
\begin{aligned}
& m=719753958, \quad n=99883476, \quad p=79929882 \quad q=39984192, \\
& r=719676954, \quad s=80045388, \quad t=99998982, \quad u=19992096
\end{aligned}
$$

or

$$
\begin{aligned}
& m=13328777, \quad n=1849694, \quad p=1480183, \quad q=740448, \\
& r=13327351, \quad s=1482322, \quad t=1851833, \quad u=370224 .
\end{aligned}
$$

Remark 4. Even though the introduced method in Section 2 provides us with the primitive solution of (2.1), methods for solving equations (3.1) and (4.1) do not provide us with primitive solutions. In these particular cases, we can find some of them using a few simple computer algorithms. For example,

$$
\begin{aligned}
(X, Y, U, V)= & (4,15,10,9),(4,16,9,7),(14,23,16,15), \\
& (20,31,14,5),(25,71,37,35), \ldots
\end{aligned}
$$

are primitive integer solutions of (3.1) and

$$
\begin{aligned}
(X, Y, U, V)= & (9,22,3,10),(10,25,2,11),(16,81,8,27), \\
& (26,73,20,27),(57,58,3,10),(62,87,21,28), \\
& (70,71,15,16),(79,92,18,25),(148,177,10,39), \ldots .
\end{aligned}
$$

are primitive integer solutions of (4.1). So, introducing a slightly different approach could be a good starting point for our further research.

\section{ACKNOWLEDGEMENT}

Both authors would like to thank Professor Andrej Dujella and the anonymous reviewer for many valuable advices and comments that improved the quality of the paper.

\section{REFERENCES}

[1] M. Baghalaghdam and F. Izadi, “A Note On The High Power Diophantine Equations," Proc. Math. Sci., vol. 129, no. 14, 2019, doi: 10.1007/s12044-018-0457-5.

[2] M. Baghalaghdam and F. Izadi, "On The Diophantine Equation in The Form That A Sum Of Cubes Equals a Sum of Quantics," Math. J. Okayama Univ, vol. 61, pp. 75-84, 2019. [Online]. Available: https://www.math.okayama-u.ac.jp/mjou/mjou61/_04_Izadi.pdf

[3] J. P. M. Binet, "Notes Sur Une Question Relative àla Thérie Des Nombres," Comptes Rendus Paris, vol. 12, pp. 248-250, 1841.

[4] A. Choudhry, "The Diophantine Equation $A^{4}+B^{4}=C^{4}+D^{4}$," Indian J. Pure Appl. Math., vol. 22, no. 1, pp. 9-11, 1991.

[5] A. Choudhry, "On The Diophantine Equation $A^{4}+h B^{4}=C^{4}+h D^{4}$," Indian J. Pure Appl. Math., vol. 26, no. 11, pp. 1057-1061, 1995.

[6] A. Choudhry, "The Diophantine Equation $a x^{5}+b y^{5}+c z^{5}=a u^{5}+b v^{5}+c w^{5}$," Rocky Mountain J. Math, vol. 29, no. 2, pp. 459-462, 1999, doi: 10.1216/rmjm/1181071645. 
[7] N. Elkies, "On $A^{4}+B^{4}+C^{4}=D^{4}$," Math. Comput., vol. 51, no. 184, pp. 825-835, 1988, doi: 10.1090/S0025-5718-1988-0930224-9.

[8] L. Euler, Novi Comm. Acad. Petrop., 6,1756-7, 155; Comm. Aerith., I, 193; Op. Om., (1), II, 428. , vol. $6,1756-7$.

[9] M. Gawron and M. Ulas, "On Primitive Integer Solutions Of The Diophantine Equation $t^{2}=$ $G(x, y, z)$ and Related Results," Journal of Number Theory, vol. 159, pp. 101-122, 2016, doi: 10.1016/j.jnt.2015.07.012.

[10] G. H. Hardy and E. M. Wright, An Introduction to the Theory of Numbers. London: Oxford University Press, 1960.

[11] F. Izadi and K. Nabardi, "Diophantine Equation $X^{4}+Y^{4}=2\left(U^{4}+V^{4}\right)$," Math. Slovaca, vol. 66, no. 3, pp. 557-560, 2016, doi: 10.1515/ms-2015-0157.

[12] A. S. Janfada and K. Nabardi, "On the Diophantine Equation $x^{4}+y^{4}=n\left(u^{4}+v^{4}\right)$," Math. Slovaca, vol. 69 , no. 6 .

[13] J. Kersey, The Elements of Algebra, London, 1674.

[14] P. Pasternak, “Zeitschr,” Math. Naturw. Unterrieht, vol. 37, pp. 33-35, 1906.

Authors' addresses

S. Bujačić Babić

Department of Mathematics, University of Rijeka, Radmile Matejčić 2, 51000 Rijeka, Croatia

E-mail address: sbujacic@math.uniri.hr

K. Nabardi

Department of Mathematics, Urmia University, P. O. Box 165, Urmia, Iran

E-mail address: k. nabardi@urmia.ac.ir 